\title{
Information Theoretic Spectral Clustering
}

\author{
Robert Jenssen ${ }^{\dagger}$, Torbjørn Eltoft ${ }^{\dagger}$ and Jose C. Principe ${ }^{\ddagger}$ \\ † Electrical Engineering Group, Department of Physics \\ University of Tromsø, Prestvannvn. 38, N-9011 Tromsø, Norway \\ $\ddagger$ Computational NeuroEngineering Laboratory, Department of Electrical and Computer Engineering \\ University of Florida, Gainesville FL. 32611, USA
}

\begin{abstract}
We discuss a new information-theoretic framework for spectral clustering that is founded on the recently introduced Information Cut. A novel spectral clustering algorithm is proposed, where the clustering solution is given as a linearly weighted combination of certain top eigenvectors of the data affinity matrix.

The Information Cut provides us with a theoretically well defined graph-spectral cost function, and also establishes a close link between spectral clustering, and non-parametric density estimation. As a result, a natural criterion for creating the data affinity matrix is provided.

We present preliminary clustering results to illustrate some of the properties of our algorithm, and we also make comparative remarks.
\end{abstract}

\section{INTRODUCTION}

A new line of research has recently emerged in the context of segmentation and clustering. It is known as the spectral clustering methods. These are methods that use the eigenvectors corresponding to certain eigenvalues (the spectrum) of a suitably chosen matrix, $\mathbf{G}$, to partition the data. Usually, $\mathbf{G}$ is the affinity matrix of the data, or a function thereof, such as the closely related Laplacian matrix.

The spectral clustering problem is often cast in graph theoretic terms. A graph consists of the node set $C$, with a symmetric similarity weight, $G_{i j} \geq 0$, corresponding to the edge between nodes $i$ and $j$. It is the matrix $\mathbf{G}=\left[G_{i j}\right]_{i, j \in C}$ that is called the affinity matrix. A graph can be bi-partitioned into two disjoint sets, or clusters, simply by removing edges between the two parts.

One measure of similarity between these two pieces can be computed as the total weight of the edges that have been removed. This quantity is called the cut. The minimum-cut criteria has been used in spectral clustering in [1]. However, the cut-cost is known to favor a skewed partition. To compensate for this fact, a number of rather heuristically motivated improvements to the cut-cost have been proposed. Specifically, we mention the normalized cut [2], the min-max cut [3], the ratio cut (average cut) [4], [5] and the foreground cut [6].

In all these methods, the bi-partitioning is performed based on a single eigenvector of the matrix $\mathrm{G}$. This spectral solution is normally obtained by formulating the cost function as a Rayleigh quotient [7], and by allowing the indicator vector,

This work was partially supported by NSF grants ECS-9900394 and EIA 0135946. or membership vector, to take real values. Normally, finding more than two clusters requires a recursive implementation of the method. Procedures for finding multi-way cuts have also been proposed [8]. Other related methods are presented in [9], [10]. For a unifying review of spectral clustering, see [11].

Despite that spectral clustering methods have been observed empirically to work well in a number of cases, the reason for their success is not well understood theoretically. For example, it is often not clear what criteria are optimized by spectral clustering. It is also not clear how to choose an edge-weight function, $G_{i j}$, such that it reflects the similarity between nodes $i$ and $j$. In the literature, $G_{i j}$ often depends on user-specified parameters. Automatic procedures for proper selection of these parameters are rarely discussed.

In this paper, we discuss a new framework for spectral clustering based on the recently introduced Information Cut (IC) [12]. In theory, clustering based on minimizing the IC leads to a high-dimensional, discrete optimization problem, which is difficult to solve. We express the IC in terms of the eigenvalues and eigenvectors of the data affinity matrix, and approximate the discrete solution by a real-valued solution. This real-valued solution is given as a linear combination of a few top eigenvectors, each weighted by the sum of its own elements. We solve the resulting low-dimensional optimization problem by developing a greedy algorithm which is able to select the right eigenvectors. To obtain the final discrete solution, we threshold the real-valued solution. There is no need to search for the best threshold, it is given by our theoretical derivations.

The main appeal of our approach is that it establishes a direct connection between graph-theoretic spectral clustering and the minimization of an information-theoretic distance measure. Furthermore, we show that spectral clustering is closely linked to non-parametric density estimation via the Parzen method, using a Gaussian kernel. As a consequence of this link, our edge-weight function is given by the Gaussian kernel. For optimum clustering performance, the width of the kernel should be chosen such that the pdf estimates are relatively accurate. This is a problem in itself, but a theoretical criterion for the selection of the kernel size may be formulated in pdf estimation.

We compare our clustering result to the clustering obtained by the foreground cut algorithm [6], since this method uses only the eigenvector corresponding to the largest eigenvalue 
of the affinity matrix in its solution. We show that this is in general not sufficient.

The organization of this paper is as follows. In section II we define the Information Cut. In section III we derive a spectral clustering algorithm based on minimizing the IC. Thereafter, in section IV, we perform some comparative clustering experiments. We give our concluding remarks in section $\mathrm{V}$.

\section{THE INFORMATION CUT}

Recently, Principe et al. [13] proposed a new informationtheoretic pdf distance measure based on the Cauchy-Schwarz (CS) inequality between two vectors. The reason for introducing this new distance measure was that it elegantly integrates non-parametric pdf estimation through Parzen windowing. This means that we can evaluate the distance measure between pdfs, without making any parametric assumptions about the underlying distributions.

Based on the Cauchy-Schwarz inequality; $\|\mathbf{x}\|^{2}\|\mathbf{y}\|^{2} \geq$ $\left(\mathbf{x}^{T} \mathbf{y}\right)^{2}$, we may write;

$$
-\log \frac{\mathbf{x}^{T} \mathbf{y}}{\sqrt{\|\mathbf{x}\|^{2}\|\mathbf{y}\|^{2}}} \geq 0 .
$$

By replacing inner products between vectors in (1), by inner products between pdfs, i.e. $\langle p, q\rangle=\int p(\mathbf{x}) q(\mathbf{x}) d \mathbf{x}$, we define the CS distance [13];

$$
D_{C S}=-\log \frac{\int p(\mathbf{x}) q(\mathbf{x}) d \mathbf{x}}{\sqrt{\int p^{2}(\mathbf{x}) d \mathbf{x}} \int q^{2}(\mathbf{x}) d \mathbf{x}} \geq 0 .
$$

In order for $D_{C S}$ to equal zero, the two pdfs must overlap completely. It goes to infinity as the overlap between the two pdfs goes to zero.

Assume that we estimate $p(\mathbf{x})$ based on the data points in cluster $C_{1}=\left\{\mathbf{x}_{i}\right\}, i=1, \ldots, N_{p}$, and $q(\mathbf{x})$ based on $C_{2}=\left\{\mathbf{x}_{j}\right\}, j=1, \ldots, N_{q}$. By the Parzen [14]. method;

$$
\begin{aligned}
& \hat{p}(\mathbf{x})=\frac{1}{N_{p}} \sum_{i=1}^{N_{p}} G\left(\mathbf{x}-\mathbf{x}_{i}, \sigma^{2} \mathbf{I}\right), \\
& \hat{q}(\mathbf{x})=\frac{1}{N_{q}} \sum_{j=1}^{N_{q}} G\left(\mathbf{x}-\mathbf{x}_{j}, \sigma^{2} \mathbf{I}\right),
\end{aligned}
$$

where we have used the multi-dimensional Gaussian kernel, $G(\mathbf{x}, \mathbf{\Sigma})$, and $\boldsymbol{\Sigma}=\sigma^{2} \mathbf{I}$. Since maximization of $D_{C S}$ is equivalent to minimization of the argument of the logarithm in (2), we now derive the expression for the latter quantity, which we denote the Information Cut (IC). This is done by substituting the pdfs by their Parzen estimates, and by utilizing the convolution theorem for Gaussians, which states that;

$$
\int G\left(\mathbf{x}-\mathbf{x}_{i}, \sigma^{2} \mathbf{I}\right) G\left(\mathbf{x}-\mathbf{x}_{j}, \sigma^{2} \mathbf{I}\right) d \mathbf{x}=G_{i j, 2 \sigma^{2} \mathbf{I}}
$$

where $G_{i j, 2 \sigma^{2} \mathbf{I}}=G\left(\mathbf{x}_{i}-\mathbf{x}_{j}, 2 \sigma^{2} \mathrm{I}\right)$. Thus, when we plug the Parzen pdf estimates of (3) into the argument of (2), and utilize (4), we obtain;

$$
\int p(\mathbf{x}) q(\mathbf{x}) d \mathbf{x} \approx \frac{1}{N_{p} N_{q}} \sum_{i, j=1}^{N_{p}, N_{q}} G_{i j, 2 \sigma^{2} \mathbf{I}}
$$

$$
\int p^{2}(\mathbf{x}) d \mathbf{x} \approx \frac{1}{N_{P}^{2}} \sum_{i, i^{\prime}=1}^{N_{p}, N_{p}} G_{i i^{\prime}, 2 \sigma^{2} \mathrm{I}}
$$

and likewise for $\int q^{2}(\mathbf{x}) d \mathbf{x}$, such that;

$$
I C=\frac{\sum_{i, j=1}^{N_{p}, N_{q}} G_{i j, 2 \sigma^{2} \mathrm{I}}}{\sqrt{\sum_{i, i^{\prime}=1}^{N_{p}, N_{p}} G_{i i^{\prime}, 2 \sigma^{2} \mathbf{I}} \sum_{j, j^{\prime}=1}^{N_{q}, N_{q}} G_{j j^{\prime}, 2 \sigma^{2} \mathbf{I}}}}
$$

If $\mathbf{x}_{i}$ and $\mathbf{x}_{j}$ are considered nodes in the set $C$, it is clear that $G_{i j, 2 \sigma^{2} \mathrm{I}}$ is in fact the similarity weight between the two nodes. This makes explicit the link between the edge-weight function and pdf estimation. It can be seen that the numerator of the Information Cut is exactly the traditional cut known from graph theory. The denominator of (7) acts as a normalizing quantity, which in our experience helps avoid the problems associated with the cut-cost alone with regard to a skewed partition. It is not motivated by heuristics, but derived from the information-theoretic origin.

Equation (7) can be written in a more compact form. We define the affinity matrix $\mathbf{G}=\left[G_{i j, 2 \sigma^{2} \mathrm{I}}\right]_{i, j \in C}$. Furthermore, we define an $N$-dimensional indicator vector, or membership vector, $\mathrm{m}$, where $N=N_{p}+N_{q}=|C|$, such that $m_{i}=1$ if node $i$ is in $C_{1}$ and 0 , if it is in $C_{2}$. Hence we obtain;

$$
I C=\frac{\mathbf{m}^{T} \mathbf{G}(\mathbf{1}-\mathbf{m})}{\sqrt{\mathbf{m}^{T} \mathbf{G m}(\mathbf{1}-\mathbf{m})^{T} \mathbf{G}(\mathbf{1}-\mathbf{m})}} .
$$

In order to perform a two-way clustering of a dataset, the goal is to determine $m$ such that the value of the Information Cut is minimized, because this corresponds to maximizing the CS distance between the two clusters. However, $m$ is a binary vector, so we are faced with a discrete minimization task, which is very difficult to solve efficiently. We take the common approach of approximating the discrete solution by allowing $m$ to take analog values, and to impose certain constraints on the solution.

\section{SPECTRAL CLUSTERING SOLUTION}

Symmetry guarantees that all of $G$ 's eigenvalues are real and that there is an orthonormal basis of eigenvectors. By the Schur decomposition [7], G can be written as;

$$
\mathbf{G}=\mathbf{E} \Lambda \mathbf{E}^{T}=\mathbf{E} \Lambda^{\frac{1}{2}} \Lambda^{\frac{1}{2}} \mathbf{E}^{T}
$$

where the columns of $\mathbf{E}$ contain the eigenvectors, and $\boldsymbol{\Lambda}=$ $\operatorname{diag}\left(\lambda_{1}, \ldots, \lambda_{N}\right)$ is a diagonal matrix that contains the corresponding eigenvalues in decreasing order.

Now we define the vector

$$
\mathbf{u}=\boldsymbol{\Lambda}^{\frac{1}{2}} \mathbf{E}^{T} \mathbf{m} \text {. }
$$

It follows that;

$$
\mathbf{\Lambda}^{\frac{1}{2}} \mathbf{E}^{T}(\mathbf{1}-\mathbf{m})=\mathbf{\Lambda}^{\frac{1}{2}} \mathbf{E}^{T} \mathbf{1}-\mathbf{\Lambda}^{\frac{1}{2}} \mathbf{E}^{T} \mathbf{m}=\mathbf{t}-\mathbf{u}
$$

where $\mathbf{t}=\Lambda^{\frac{1}{2}} \mathbf{E}^{T} \mathbf{1}$ and $\mathbf{1}$ is a $(N \times 1)$ vector whose elements are all one. By inserting (9) into (8), and utilizing (10) and (11), we obtain the following expression for the IC;

$$
I C=\frac{\mathbf{u}^{T}(\mathbf{t}-\mathbf{u})}{\sqrt{\|\mathbf{u}\|^{2}\|\mathbf{t}-\mathbf{u}\|^{2}}}=\cos \angle(\mathbf{u}, \mathbf{t}-\mathbf{u}) .
$$


We proceed by seeking the $\mathbf{u}$ that satisfies;

$$
\min _{\mathbf{u}} I C=\min _{\mathbf{u}} \cos \angle(\mathbf{u}, \mathbf{t}-\mathbf{u}) .
$$

In order to be able to utilize (13) we need to obtain some constraints on $\mathbf{u}$. We know that for any discrete solution, the following holds: $\mathbf{m}^{T}(\mathbf{1}-\mathbf{m})=0$. This is equivalent to the following constraint on $\mathbf{u}$;

$$
\mathbf{u}^{T} \boldsymbol{\Lambda}^{-1}(\mathbf{t}-\mathbf{u})=0
$$

which in fact is the equation of a hyper-ellipse in the $N$ dimensional space. Hence, we seek the $\mathbf{u}$ that lies on the hyperellipse described by (14), and at the same time minimizes the IC.

Now, notice that any vector $\mathbf{u}$ of the form;

$$
u_{i} \in\left\{t_{i}, 0\right\}, i=1, \ldots, N
$$

does indeed obey (14). Furthermore, it has the property that;

$$
\mathbf{u} \perp \mathbf{t}-\mathbf{u} \Rightarrow \cos \angle(\mathbf{u}, \mathbf{t}-\mathbf{u})=0
$$

which means that such a vector minimizes the Information Cut.

Remember that $\mathbf{u}=\Lambda^{\frac{1}{2}} \mathbf{E}^{T} \mathrm{~m}$, such that;

$$
\mathbf{m}=\Lambda^{-\frac{1}{2}} \mathbf{E u}=\sum_{i=1}^{N} \frac{u_{i}}{\sqrt{\lambda_{i}}} \mathbf{e}_{i}
$$

where $\mathbf{e}_{i}$ is the $i$ th column of $\mathbf{E}$, i.e. the $i$ 'th eigenvector of $\mathbf{G}$. Define $w_{i}=1$ if $u_{i}=t_{i}$, and $w_{i}=0$ if $u_{i}=0, i=1, \ldots, N$. Thus, the discrete solution can be written;

$$
\mathbf{m}=\sum_{i=1}^{N} w_{i} \frac{t_{i}}{\sqrt{\lambda_{i}}} \mathbf{e}_{i}=\sum_{i=1}^{N} w_{i}\left(\mathbf{e}_{i}^{T} \mathbf{1}\right) \mathbf{e}_{i},
$$

since $t_{i}=\sqrt{\lambda_{i}} \mathbf{e}_{i}^{T} \mathbf{1}$. In conclusion, our solution is given as a linearly weighted summation of some of the eigenvectors, where the weighting on each eigenvector is given by the sum of the elements of that eigenvector.

Now we need to determine which eigenvectors to use, such that (18) is indeed the correct discrete solution. Of course, $\mathbf{u}=\mathbf{0}$ or $\mathbf{u}=\mathbf{t}$ are not valid solutions, since these solutions translate into $\mathbf{m}=\mathbf{0}$ or $\mathbf{m}=\mathbf{1}$. These are trivial solutions, meaning that all data points are assigned to only one cluster.

\section{A. The real-valued solution: reducing complexity}

We are faced with the problem of finding some $N$ dimensional vector $\mathbf{u}$ of the form (15). Any such vector will minimize the IC, which makes this a high-dimensional optimization problem. Fortunately, it turns out that most of the elements of the vector $\mathbf{t}$ are typically very close to zero, a fact that we have observed experimentally on a number of datasets. There are in fact only a small number $M$ of such elements, where $M \ll N$, deviating significantly from zero. We embed our optimization problem in the $M$-dimensional real-value domain, by relaxing the discrete vector $m$ to take analog values, and proceed in order to determine a vector $\hat{\mathbf{u}}$, such that $\hat{u}_{i}=t_{i}$ or $\hat{u}_{i}=0$, for $i=1, \ldots, M$. Hence $\hat{\mathbf{m}}=\boldsymbol{\Lambda}^{-\frac{1}{2}} \mathbf{E} \hat{\mathbf{u}}$.

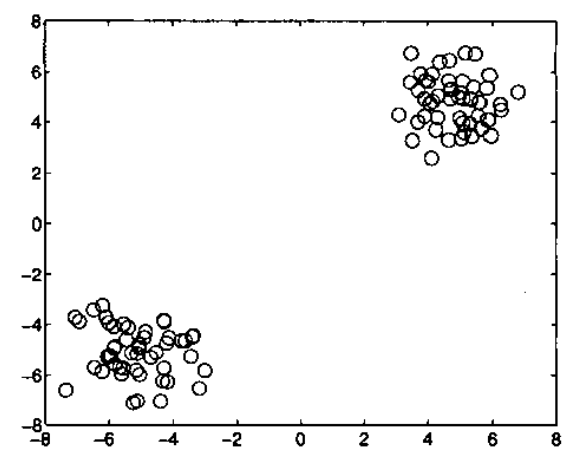

(a)
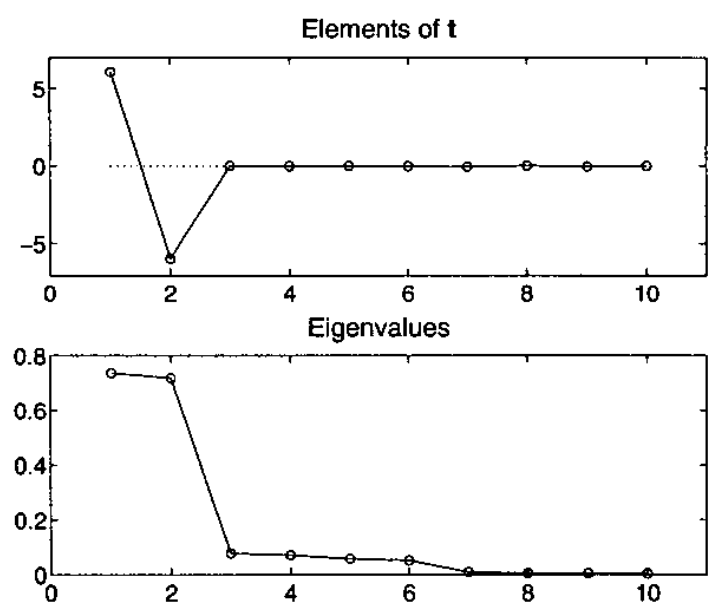

(b)

Fig. 1. Dataset consisting of two Gaussian clusters, shown in (a). Elements of $t$ and $\Lambda$ are shown in (b).

To obtain the discrete solution, $\mathbf{m}$, we threshold $\hat{\mathbf{m}}$ such that elements larger than $1 / 2$ are given the value one, and elements smaller than $1 / 2$ are given the value zero.

Of course, the number of significantly large elements of $t$ varies from dataset to dataset, and is also closely dependent on the edge-weight function used, in our case it depends on the parameter $\sigma$. In our experience, one reason for this behaviour of $t$ is that there are typically only a few of the eigenvalues of the affinity matrix that deviate significantly from zero. Since $t_{i}=\sqrt{\lambda_{i}} \mathbf{e}_{i}^{T} \mathbf{1}$, it will be close to zero if $\lambda_{i}$ is close to zero. This is true since $\mathbf{E}$ is orthonormal, hence the sum of the elements of each eigenvector is bounded, since $\mathbf{e}_{i}^{T} \mathbf{e}_{i}=1$. Another reason is that frequently $\mathbf{e}_{i}^{T} 1 \approx 0$, such that $t_{i}$ may also be very close to zero even if $\lambda_{i}$ is not.

As an example, consider the simple dataset shown in Fig. 1 (a). For a suitably chosen $\sigma$, only two of the elements of $t$ deviate significantly from zero. This is illustrated in the upper panel of Fig. 1 (b), where the ten first entries of $t$ have been shown for $\sigma=2.14$. We will return to the selection of $\sigma$ in section III-C. In fact, for this dataset, there are also only two 


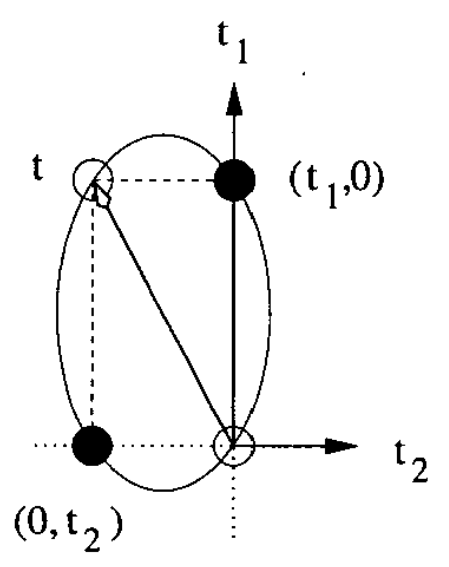

(a)

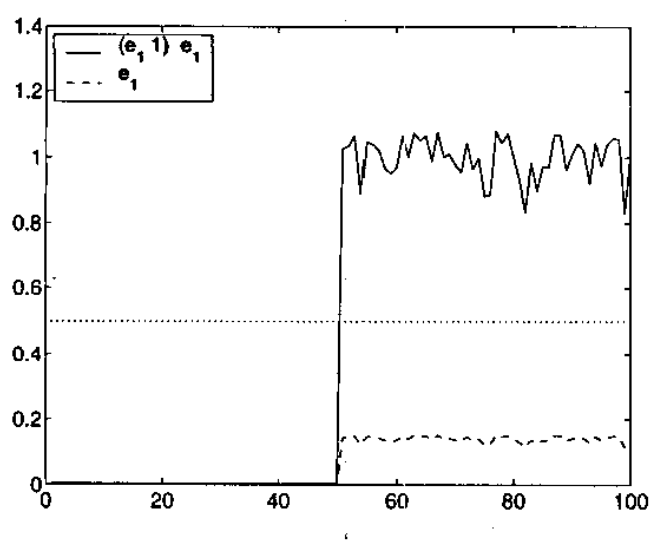

(b)

Fig. 2. The solution ellipse (14) is shown in (a). The real-valued solution $\hat{\mathbf{m}}$ and the largest eigenvector $e_{1}$ is shown in (b). The horizontal line (dotted) indicates the threshold at $1 / 2$.

significant eigenvalues. This is illustrated in the lower panel of Fig. 1 (b), where the first ten entries on the diagonal of $\Lambda$ have been shown.

Fig. 2 (a) shows the ellipse described by (14) for $\mathbf{t}=$ $\left[t_{1}, t_{2}\right]^{T}$. The IC is minimized by selecting either $\hat{\mathbf{u}}=\left[t_{1}, 0\right]^{T}$ or $\hat{\mathbf{u}}=\left[0, t_{2}\right]^{T}$, since it follows that $\hat{\mathbf{u}} \perp \mathbf{t}-\hat{\mathbf{u}}$. These two solutions are equivalent; they both divide the graph into the same two groups, but they give opposite labels to the two clusters. Let us examine the solution based on $\hat{\mathbf{u}}=\left[t_{1}, 0\right]^{T}$, that is, $\hat{\mathbf{m}}=\left(\mathbf{e}_{1}^{T} \mathbf{1}\right) \mathbf{e}_{1}$. In Fig. 2 (b) we show a plot of both $\hat{\mathbf{m}}$ and $\mathbf{e}_{1}$. The dataset has been ordered, such that the first 50 elements of these vectors correspond to the cluster to the left, and the last 50 correspond to the other cluster. Clearly, $\hat{\mathbf{m}}$ approximates the discrete solution very closely. We conclude that our information-theoretic approach has revealed that when there are two significant elements of $t$, then there are two equivalent solutions, namely either $\hat{\mathbf{m}}=\left(\mathbf{e}_{1}^{T} \mathbf{1}\right) \mathbf{e}_{1}$ or $\hat{\mathbf{m}}=\left(\mathbf{e}_{2}^{T} \mathbf{1}\right) \mathbf{e}_{2}$.

Note that the foreground cut algorithm [6] uses the eigenvector corresponding to the largest eigenvalue to partition the graph. Hence, for a dataset such as the one described above, we expect our algorithm and the foreground cut algorithm to obtain similar clustering results. However, when using the foreground cut, it is not clear how to choose the threshold. Fig. 2 (b) clearly shows that in this case there exists a threshold that yields the same result as that given by the IC.

In general, however, there is no guarantee that there will be only two significant elements of $t$. In such cases the foreground cut algorithm will fail.

In the following we briefly describe our new information theoretic spectral clustering algorithm.

\section{B. The spectral algorithm}

The main idea behind our algorithm is very simple. We take as our starting point that the eigenvector $e_{1}$, corresponding to $t_{1}$, is a part of the sum (18) that constitutes the solution. One-by-one, we include temporarily the other eigenvectors corresponding to the remaining $t_{i}, i=2, \ldots, M$, into the sum. In each case the IC is calculated. We pick the next eigenvector to be appended permanently, to be the component that yields the smallest IC-value. This procedure is terminated if the IC-value increases from one iteration to the next. A pseudo-code for this algorithm is given below. We denote by $\mathbf{e}^{\prime}$ an eigenvector that has not yet been included in the solution.

- Eigendecompose G

- Calculate t

- $M=\#$ significant $t_{i}^{\prime}$ s

- Initialize: $\hat{\mathbf{m}}=\left(\mathbf{e}_{1}^{T} \mathbf{1}\right) \mathbf{e}_{\mathbf{1}}$

- Determine $I C(\mathbf{m})$

- while loop

$$
\begin{aligned}
& \text { - for } j=1: \mathrm{M}-1 \\
& \quad \hat{\mathbf{m}}_{j}=\hat{\mathbf{m}}+\left(\mathbf{e}_{j}^{\prime T} \mathbf{1}\right) \mathbf{e}_{j}^{\prime} \\
& \quad \text { Store } I C\left(\mathbf{m}_{j}\right) \\
& \text { end for } \\
& \text { - Find } \hat{\mathbf{m}}_{\min }: \min _{\hat{\mathbf{m}}_{j}} I C\left(\mathbf{m}_{j}\right), j=1, \ldots, M-1 \\
& \text { - if } I C\left(\mathbf{m}_{\min }\right)<I C(\mathbf{m}) \\
& \quad \hat{\mathbf{m}}=\hat{\mathbf{m}}_{\min } \\
& \quad M=M-1 \\
& \text { else stop } \\
& \text { end if }
\end{aligned}
$$

until stop

The number $M$ of significant elements of $\mathrm{t}$ is determined by selecting the elements whose value is larger than $0.1 \times$ $t_{i, \max }, i=1, \ldots, N$. In order to cluster the data into several groups, we apply this spectral algorithm recursively.

\section{Creating the affinity matrix}

A major drawback of the vast majority of the existing spectral clustering algorithms, is that it is not clear how to choose an edge-weight function in order to construct the affinity matrix. In many cases, the Gaussian edge-weight function is used, which requires the user to specify the width, $\sigma$, of the Gaussian kernel. It has been observed that the results obtained by various algorithms may vary significantly depending on $\sigma$. This also makes a fair comparison between different algorithms difficult. 
Using the Information Cut, $\sigma$ is in fact closely tied to Parzen pdf estimation. Selecting a proper kernel size for Parzen pdf estimation is by no means a trivial task, especially for highdimensional data. Still, we will show that we are able to obtain promising preliminary clustering results using our Information Cut algorithm, where the affinity matrix is automatically created. Here, we briefly state Silverman's [15] "rule-of-thumb" for automatic selection of the "optimal" kernel size for Parzen pdf estimation.

The mean integrated square error (MISE) is the most widely used measure on the global accuracy of an estimator $\hat{p}(\mathbf{x})$ of the pdf $p(\mathbf{x})$

$$
\operatorname{MISE}(\hat{p})=E \int\{\hat{p}(\mathbf{x})-p(\mathbf{x})\}^{2} d \mathbf{x}
$$

The MISE can be approximated asymptotically, from which an expression for the optimal kernel size, $\sigma_{\text {opt }}$, can be derived. The problem is that $\sigma_{\text {opt }}$ itself depends on the second derivative of the density we wish to estimate. The "rule-of-thumb" approach is to obtain a rough estimate of the unknown quantity by assuming that the underlying density is Gaussian. This rough estimate is then plugged back into the expression for $\sigma_{\text {opt }}$. Hence, $\sigma_{\text {opt }}$ will only be optimal for a Gaussian density, and will normally result in a over-smoothed estimate for nonGaussian data.

For $d$-dimensional data, the "optimal" kernel size is given by [15];

$$
\sigma_{\mathrm{op} t}=s\left(\frac{4}{N(2 d+1)}\right)^{\frac{1}{d+4}},
$$

where $s^{2}=d^{-1} \sum_{i} S_{i i}$, and $S_{i i}$ are the diagonal elements of the sample covariance matrix. In our experiments, we have utilized $(20)$ in order to create the affinity matrix. However, for strongly non-Gaussian datasets it has a tendency to oversmooth. We therefore suggest to estimate the optimal onedimensional kernel size for each dimension of the data, and use the smallest such value as our $\sigma$. The one-dimensional "optimal" bandwidth is given by [15];

$$
\sigma_{\text {opt }}=s 1.06 N^{-1 / 5},
$$

where $s$ is an estimate of the standard deviation of the onedimensional data.

In all examples and clustering experiments in this paper, the data affinity matrix is automatically created based on (21) and the above discussion.

\section{CluSTERING EXPERIMENTS}

In the first experiment we consider a dataset consisting of a concentric ring with a Gaussian cluster in the middle. We provide the dataset only to our algorithm, there are no userspecified parameters. The output is shown in Fig. 3 (a), which is in fact the correct solution.

In Fig. 3 (b), the first ten entries of $t$, and of the diagonal of $\boldsymbol{\Lambda}$, are shown in the upper and lower panels, respectively. The algorithm selects three significant elements of $t$, corresponding to the eigenvalues $\lambda_{1}, \lambda_{2}$ and $\lambda_{4}$. Note that all displayed eigenvalues have fairly large values, as opposed to the previous example.

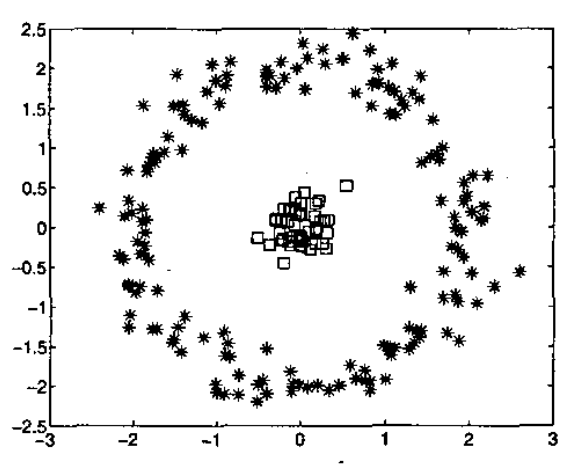

(a)
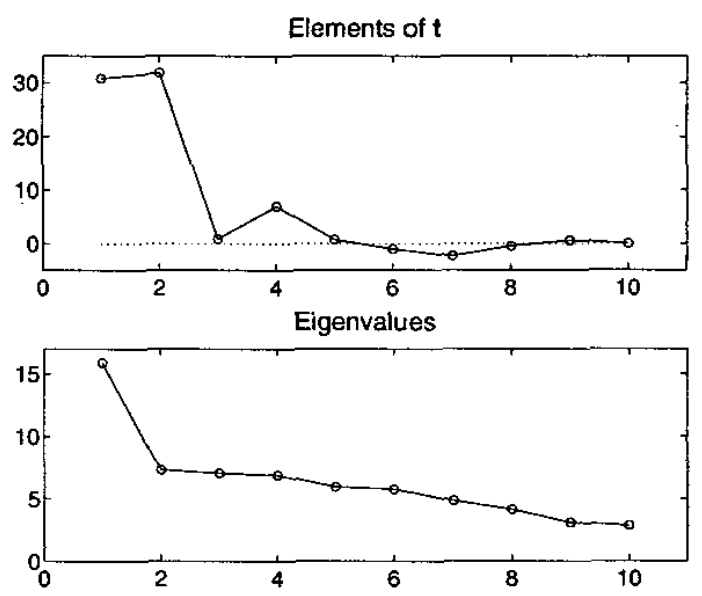

(b)

Fig. 3. Dataset consisting of a concentric ring with a Gaussian cluster in the middle. The correct clustering is shown in (a), while elements of $\mathbf{t}$ and $\Lambda$ are shown in (b).

The algorithm determines the solution to be $\hat{\mathbf{m}}=\left(\mathbf{e}_{1} \mathbf{1}\right) \mathbf{e}_{1}$, and the opposite solution (switching labels) to be given by $\left(\mathbf{e}_{2} \mathbf{1}\right) \mathbf{e}_{2}+\left(\mathbf{e}_{4} \mathbf{1}\right) \mathbf{e}_{4}$. Hence, also in this case the foreground cut algorithm should work well since a solution based on the largest eigenvector can be found. This is indeed the case, using the same $\sigma$ as our algorithm determined.

In the second experiment we consider a dataset consisting of two equally sized half-circles. The output of the algorithm is shown in Fig. 4 (a). The clustering is completely correct.

Fig. 4 (b) shows that there are seven significant elements of t. All displayed eigenvalues are large, but decreasing slowly in value. The algorithm determines that clustering based on the largest eigenvector alone is not sufficient for this dataset. It includes another two eigenvectors in the sum (18).

Fig. 4 (c) shows a plot of the largest eigenvector, $\mathbf{e}_{1}$. Also in this case the dataset has been ordered, such that the first half of the elements of $e_{1}$ correspond to the cluster to the left, and the second half to the cluster to the right. There is no way to find a threshold resulting in a perfect clustering. In this case, 
the foreground cut algorithm fails.

\section{CONCLUSIONS}

We have discussed a new information-theoretic framework for spectral clustering based on the Information Cut.

We have developed a novel spectral clustering algorithm, where the real-valued clustering solution is given as a linearly weighted combination of a few of the top eigenvectors, where the weighting on each eigenvector is given by the sum of the elements of that vector. This algorithm is fully automatic, since there are no user-specified parameters needed in order to construct the affinity matrix. This is a major advantage compared to other spectral methods in the literature.

Another advantage is that our spectral clustering cost function is well defined, originating from an informationtheoretic pdf distance measure based on the Cauchy-Schwarz inequality. Thus, an interesting link between graph-theory and information-theory is revealed, which also shows that spectral clustering is closely related to non-parametric pdf estimation.

In our future work, we will further study the issue of automatic selection of a proper kernel size for relatively accurate Parzen density estimation. This may be of special importance when it comes to high-dimensional data.

\section{REFERENCES}

[1] N. Cristianini, J. Shawe-Taylor, and J. Kandola, 'Spectral Kernel Methods for Clustering," in Advances in Neural Information Processing Systems, 14, 2001, vol. 1, pp. 649-655.

[2] J. Shi and J. Malik, 'Normalized Cuts and Image Segmentation," IEEE Transactions on Pattern Analysis and Machine Intelligence, vol. 22, no. 8, pp. 888-905, 2000.

[3] C. H. Q. Ding, X. He, H. Zha, M. Gu, and H. D. Simon, "A Min-max Cut Algorithm for Graph Partitioning and Data Clustering," in IEEE Int. Conf. on Data Mining, 2001, pp. 107-114.

[4] L. Hagen and A. B. Kahng, 'Fast Spectral Methods for Ratio Cut Partitioning and Clustering," in International Conference on ComputerAided Design, Santa Clara, CA, USA, 1991, pp. 10-13.

[5] S. Sarkar and P. Soundararajan, 'Supervised Learning of Large Perceptual Organization: Graph Spectral Partitioning and Learning Automata," IEEE Transactions on Pattern Analysis and Machine Intelligence, vol. 22, no. 5, pp. 504-525, 2000 .

[6] P. Perona and W. T. Freeman, "A Factorization Approach to Grouping," in Proc. European Conference on Computer Vision, 1998, pp. 655-670.

[7] G. H. Golub and C. F. Van Loan, Matrix Computations, The John Hopkins University Press, 1996.

[8] M. Meila and L. Xu, 'Multiway Cuts and Spectral Clustering," Tech. Rep. 442, University of Washington, Deartment of Statistics, January 2004.

[9] A. Y. Ng, M. Jordan, and Y. Weiss, "On Spectral Clustering: Analysis and an Algorithm," in Advances in Neural Information Processing Systems, I4, 2001, vol. 2, pp. 849-856.

[10] R. Kannan, S. Vempala, and A. Vetta, 'On Clusterings: Good, Bad and Spectral," in IEEE Foundations of Computer Science, Redondo Beach, CA, USA, 2000, pp. 367-377.

[11] Y. Weiss, 'Segmentation Using Eigenvectors: A Unifying View," in International Conference on Computer Vision, 1999, pp. 975-982.

[12] R. Jenssen, J. C. Principe, and T. Eltoft, "Information Cut and Information Forces for Clustering," in IEEE International Workshop on Neural Networks for Signal Processing, Toulouse, France, 2003, pp. $459-468$.

[13] J. Principe, D. Xu, and J. Fisher, "Information Theoretic Learning," in Unsupervised Adaptive Filtering, S. Haykin (Ed.), John Wiley \& Sons, 2000 , vol. I, Chapter 7.

[14] E. Parzen, "On the Estimation of a Probability Density Function and the Mode," Ann. Math. Stat., vol. 32, pp. 1065-1076, 1962.

[15] B. W. Silverman, Density Estimation for Statistics and Data Analysis, Chapman and Hall, 1986.

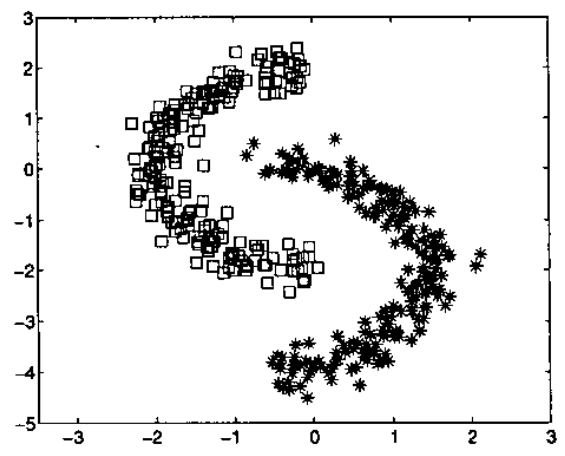

(a)
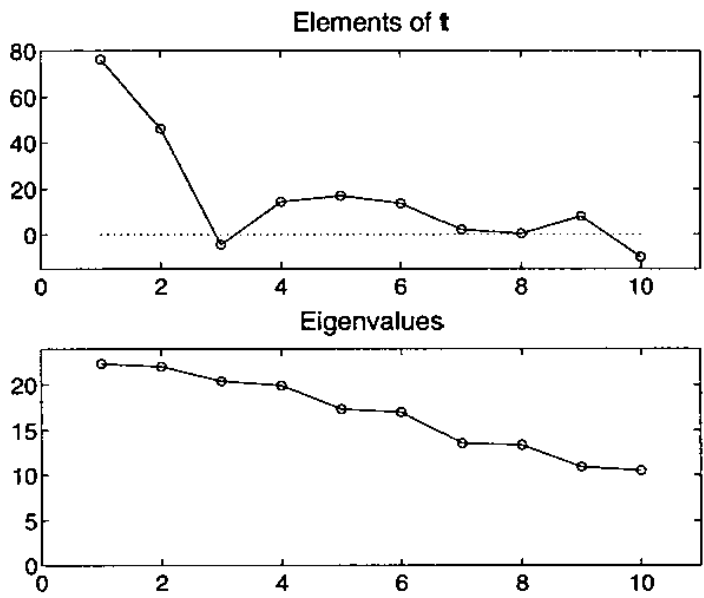

(b)

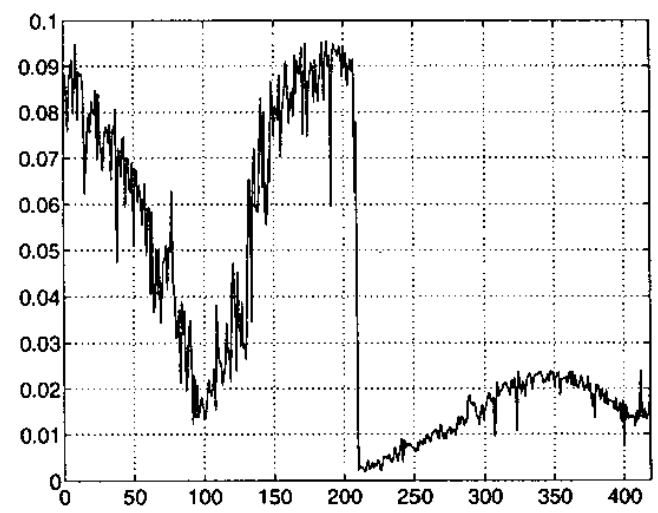

(c)

Fig. 4. Dataset consisting of two half-circles. The correct clustering is shown in (a), while elements of $\mathbf{t}$ and $\boldsymbol{\Lambda}$ are shown in (b). 